\title{
Marco de trabajo de Cobit para en el adecuado uso de tecnologías de información en organizaciones públicas y privadas
}

\author{
Cobit framework for the adequate use of information technologies in public and \\ private organizations \\ ${ }^{1}$ Henry George Maquera Quispe \\ ORCID: 0000-0003-3055-9166 \\ ${ }^{2}$ Marjorie Gabriela del Carmen Delgado Rospigliosi \\ ORCID: 0000-0001-9217-6449 \\ ${ }^{3}$ María Elena Tasa Catanzaro \\ ORCID: 0000-0003-0106-6311
}

\section{RESUMEN}

El trabajo de investigación se basó en el análisis del cambio organizacional bajo la implementación del marco de trabajo de Cobit, para garantizar el correcto uso de los activos de tecnologías de información. La investigación se llevó a cabo en organizaciones públicas y privadas de la región central del Perú. Se contó con la participación del personal asignado en las áreas de tecnologías de información, que permitieron el acceso a información respecto a los procesos y servicios de tecnologías de información de las organizaciones bajo estudio. El método utilizado fue el analítico debido a que se observó causas y efectos partiendo de los principios de las buenas prácticas de Cobit. La investigación permitió establecer que existe una directa y sólida correlación entre la percepción de los trabajadores y el uso del marco de trabajo de Cobit. Los resultados indicaron que la participación de buenas prácticas de ITIL permitieron una mejor integración de los activos de TI en el adecuado desarrollo del marco de trabajo de Cobit. Sin embargo, se apreció una diferenciada evolución entre las organizaciones públicas y privadas, mayormente, debido a factores internos como la cultura organizacional existente.

Palabras clave: Cobit, gobierno, ITIL, servicio

\begin{abstract}
The research work is based on the analysis of organizational change under the implementation of the Cobit framework to ensure the correct use of information technology assets. The research was carried out in public and private organizations in the central region of Peru. There was the participation of assigned personnel in the areas of information technologies that allowed access to information regarding the information technology processes and services of the organizations under study. The method used was analytical because causes and effects were observed based on the principles of good Cobit practices. The research allowed us to establish that there is a direct and solid correlation between the perception of workers and the use of the Cobit framework. The results indicated that the participation of ITIL good practices allowed a better integration of IT assets in the proper development of the Cobit framework. However, there was a different evolution between public and private organizations, mainly due to internal factors such as the existing organizational culture.
\end{abstract}

Keywords: Cobit, government, ITIL, service.

Facultad de Ingeniería de Sistemas. Universidad Nacional del Centro del Perú. Huancayo - Perú. E-mail: hmaquera@uncp.edu.pe

${ }^{2}$ Facultad de Ingeniería de Sistemas. Universidad Nacional del Centro del Perú. Huancayo - Perú. E-mail: delgado.mc@pucp.edu.pe

Facultad de Ingeniería Electrónica y Sistemas. Universidad Nacional de Huancavelica. Huancavelica - Perú. E-mail: maria.tasa@unh.edu.pe

Nota: El presente artículo es derivado del proyecto de investigación "Buenas prácticas de gobierno corporativo de Tecnologías de Información en organizaciones públicas y privadas", presentado por el Grupo de Investigación de la Facultad de ingeniería de Sistemas de la Universidad Nacional del Centro del Perú. 


\section{INTRODUCCIÓN}

La dirección de Tecnologías de Información (en adelante TI), tiene bajo su responsabilidad proporcionar servicios a diversas áreas de una organización. Sin embargo, diversas organizaciones no logran mejorar la calidad de los servicios de TI a través de la aplicación de un marco de trabajo adecuado para el control y supervisión. Esto se debe a que no se cuenta con un diagnóstico del estado de los servicios de TI proporcionados y se carece de una evaluación de las mejoras logradas a través del uso de los servicios de TI en las organizaciones, que trae consigo altos niveles de incertidumbre en las estrategias que se deban implementar para mejorar la calidad de servicios de TI. Por lo que la investigación se fundamentará en la percepción del personal que utiliza los servicios críticos de TI en relación a las mejoras logradas a través del marco de trabajo de Cobit (Control Objectives for Information and related Technology, en adelante Cobit) en organizaciones públicas y privadas bajo la participación de un diagnóstico de los servicios de TI y la evolución del uso del marco de trabajo de Cobit por medio de la aplicación de un modelo de estimación basado en la mejora, evaluación de procesos de desarrollo e implementación de soluciones de TI.

Existe una relación directa y fuerte entre la percepción de los trabajadores y el uso del marco de trabajo de Cobit. Los usuarios internos y externos a la organización perciben esto basándose en la ejecución de los procesos y servicios de tecnologías de información más agiles, más controlados y encaminados en relación con las políticas de la organización. De igual manera, las herramientas de recolección de información deben ser actualizadas y revisadas periódicamente, a fin de garantizar la mejor fuente de información.

Para este efecto, dentro del proceso de evaluación del funcionamiento de las soluciones de tecnologías de información en la organización es posible utilizar el Control de Objetivos para Tecnologías de Información y Relacionadas, debido a que alinea los procesos formalizados por la organización con los factores de riesgo y procede a generar información de los mismos con el fin de mejorar los procesos de toma de decisión (Astuti, Muqtadiroh, Darmaningrat, \& Putri, 2017). La participación de los procesos establecidos en una organización se han transformado debido a que el gobierno corporativo de TI guarda una relación directa con los niveles de madurez de los procesos establecidos dentro de la organización (Joshi,
Bollen, Hassink, De Haes, \& Van Grembergen, 2018).

Las organizaciones, hoy en día, dependen altamente de la información generada por el uso de soluciones de TI. La implementación de un gobierno corporativo de TI en una organización es un factor desafiante dentro de la misma, debido a la existencia de un amplio espacio entre conocimientos teóricos y conocimientos pragmáticos (Smits \& van Hillegersberg, 2018). La información generada se transforma luego en activos que son cada vez más vitales a considerar dependiendo del tipo de organización. Las organizaciones cada vez más competitivas tienden a implementar mecanismos operativos formales de manera que sea apreciable la generación de valor de las herramientas de TI. Sin embargo, el impacto de la gobernanza de TI no se aprecia en factores tales como el apoyo de la alta gerencia, desempeño del comité directivo de Tecnologías de Información o en la planificación estratégica(Santos \& Santos, 2017).

\section{Trabajos relacionados}

El gobierno corporativo de tecnologías de información ha permitido a diversas empresas alcanzar el éxito. Cobit permite desarrollar el tratamiento de soluciones de TI bajo un enfoque de riesgos y valor, lo cual nos faculta a guiar la implementación de un sistema integral con el fin de minimizar los riesgos asociados y optimizar el valor (Alreemy, Chang, Walters, \& Wills, 2016). Las organizaciones son conocedoras de este nuevo escenario, por lo que cada vez van mejorando su ambiente tecnológico relacionado con infraestructura al mismo tiempo que incorporan mejores soluciones de seguridad de la información. Esta combinación de tecnológica ha obligado a lograr establecer un mejor mecanismo de organización y gobierno relacionado a tecnologías de información, con el fin de alinear la evolución tecnológica con la política de la organización.

Sin embargo, la efectividad del gobierno de TI se ve afectada por los mecanismos no operativos establecidos en la organización (Santos \& Santos, 2017), es por ello que se llega a estudiar las áreas de supervisión, planificación, implementación, evaluación en los diversos productos tecnológicos implementados. Si bien se considera a la información como un recurso muy importante en la organización, también el software es considerado como uno de los activos de tecnologías de información más utilizados en organizaciones. Las herramientas de comunicación entre el cliente y el proveedor usadas en el desarrollo de un proyecto de software se ven afectadas por funciones de control de TI 
establecidas por Cobit (Gantman \& Fedorowicz, 2016).

Las organizaciones han empezado a atravesar un proceso evolutivo en la conformación de una estructura de gestión y gobierno con el fin de garantizar el logro de metas corporativas en la planificación y ejecución de actividades alineadas con la dirección establecida, y para ello el uso de terminología adecuada ayuda a consolidar conocimientos y proporciona instrucciones para el logro de las metas esperadas (Huse, 2005). Asimismo, la agilidad empresarial se ha convertido en una de las principales preocupaciones organizativas más importantes para la administración de TI. Para alinear las metas de la organización con las tecnologías de la información se debe establecer el uso efectivo y eficiente de los sistemas de información y la tecnología de la información, es decir, la gobernanza de tecnologías de información (Sá Couto, Filomena Castro Lopes, \& Dinis Sousa, 2015).

Las organizaciones en la ciudad de Huancayo vienen utilizando TI en los diversos procesos establecidos entre sus unidades de negocio. Las organizaciones vienen dependiendo cada vez más de implementaciones de tecnologías de información que agilizan procesos y reducen niveles de riesgo en el tratamiento de la información. Por lo que Cobit introduce estrategias que permiten a las tecnologías de información ser gestionadas y gobernadas de un modo holístico a lo largo de toda la organización. Esta nueva estructura de gobierno y gestión abarca el negocio en su totalidad y ofrece una visión de trabajo para todo tipo de empresa. Por otro lado, la norma ISO / IEC 38500 ha permitido a múltiples organizaciones implementar los marcos de gobierno de tecnologías de información adecuados (Juiz, Colomo-Palacios, \& Gómez, 2018).

Las organizaciones analizadas cuentan con un departamento de TI, que viene implementando mecanismos de mejora continua a través de la ejecución de proyectos. Sin embargo, estas organizaciones no cuentan con una guía formal para llevar a cabo estos proyectos, debido a que la ejecución de los mismos se fundamenta en decisiones prácticas más que en decisiones fundamentadas en algún análisis formal. Se ha descuidado la oportunidad que brinda Cobit en asegurar que las organizaciones administren sus actividades relacionadas con TI de un modo aceptable y confiable(Isaca, 2012b) a través de la aplicación de sus principios clave:

- Principio 1. Satisfacer las necesidades de las partes interesadas.
- Principio 2: Cubrir la empresa extremo-aextremo.

- Principio 3: Aplicar un marco de referencia único integrado.

- Principio 4: Hacer posible un enfoque holístico.

- Principio 5: Separar el gobierno de la gestión.

\section{MATERIALY MÉTODOS}

El estudio se ha dirigido a empresas medianas y grandes que cuenten con un departamento de TI, por lo que la población de estudio se fijó en los procesos de tecnologías de información de 37 organizaciones medianas y grandes en la región central del Perú. Se estableció una muestra dirigida a los recursos y capacidades de TI de diez organizaciones, de las cuales cinco son de naturaleza privada y cinco son de naturaleza pública. La muestra dirigida tuvo como característica a organizaciones que permitieron el completo acceso a la información requerida para el estudio y los activos, el estudio estuvo fundamentada en las buenas prácticas de ITIL. La Biblioteca de Infraestructura de TI ( en adelante ITIL) es uno de los marcos de gobierno de TI, que brinda soporte a la organización para administrar los servicios de TI de manera efectiva (Gërvalla, Preniqi \& Kopacek, 2018). La Figura 1 resume la participación de capacidades y recursos de TI que una organización contempla como activos. El activo considerado como recurso y capacidad en forma simultanea son las personas, por lo que se consideró a las 59 personas de organizaciones públicas y a las 95 personas de organizaciones privadas que laboran en el área de TI.

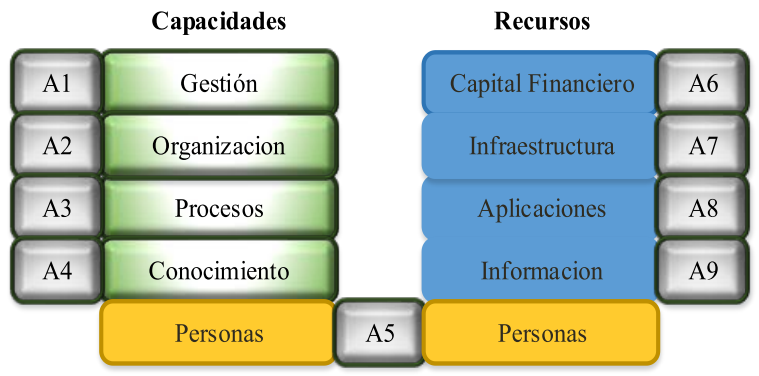

Figura 1. Capacidades y Recursos de TI en una organización.

En el estudio se utilizó listas de comprobación y cuestionarios como técnicas de análisis cuantitativo, asociados a los 37 procesos de gobierno y gestión de Cobit. La relación entre las técnicas de análisis cuantitativo y procesos de Cobit se basó en el principio de servicios establecidos por ITIL debido a que la gestión y el uso de los servicios de TI se consideran muy importantes para que las empresas modernas 
mejoren su rendimiento(Gërvalla et al., 2018). La Figura 2 resume la relación entre los diferentes componentes de una organización y su interrelación con los componentes de TI.

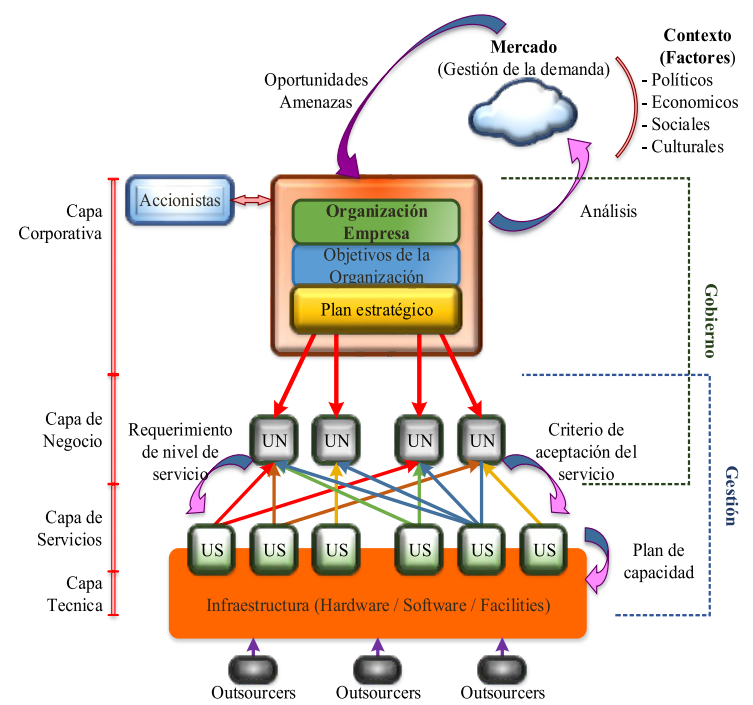

Figura 2. Interrelación entre componentes de una organización basado en Servicios de TI.

La Figura 2 muestra el conjunto completo de los procesos de acuerdo al marco de trabajo del modelo Cobit. Este marco de trabajo proporciona una guía de análisis por cada proceso y dominio establecido e información respecto al gobierno con los procesos EDM-Evaluar, Orientar y Supervisar.

Mientras que los dominios de procesos de gestión se establecen en:
APO - Alinear, Planificar y Organizar.

BAI-Construir, Adquirir e Implementar.

DSS - Entrega, Servicio y Soporte.

MEA-Supervisar, Evaluar y Valorar.

La Figura 3 resume la interacción entre los diversos procesos establecidos por Cobit para garantizar el adecuado gobierno de tecnologías de información que permita interrelacionar el riesgo de TI como un riesgo de negocio, específicamente, el riesgo de negocio asociado con el uso, propiedad, operación, participación, influencia y adopción de TI dentro de una empresa (Astuti et al., 2017). Cobit ha proporcionado un modelo de referencia que representa todos los procesos que normalmente se encuentran en una empresa en relación con las actividades de TI, y ofrece un modelo de referencia común comprensible para los gerentes de negocios y de TI operativos. El modelo de proceso propuesto es un modelo completo e integral, pero no es el único modelo de proceso posible.

La investigación se inició con la aplicación de las listas de comprobación en los servicios y procesos que consumen activos de TI establecidos en las organizaciones. Los puntos considerados en la lista de comprobación se basaron en:

- Continuidad de servicios de TI.

- Cumplimiento legal.

- Clasificación de la información.

- Aplicaciones permitidas.

- Almacenamiento en la red corporativa.

- Gestión de recursos humanos.

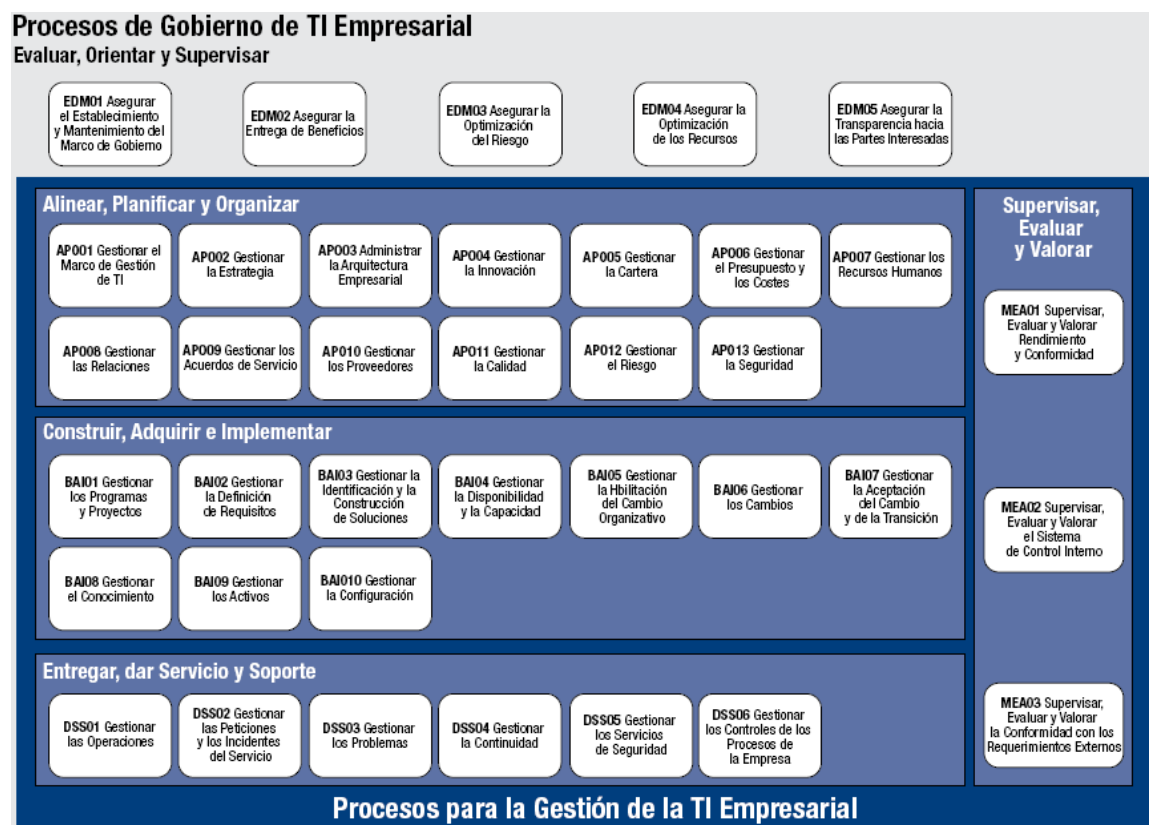

Figura 3. Modelo de referencia de procesos de Cobit (Isaca, 2012b). 
La Tabla 1 presenta la escala utilizada para la evaluación del estado de los servicios en producción de las organizaciones, se basó en una arquitectura de dos dimensiones fundamentada en los niveles de capacidad y atributos propuesto por la ISO/EIC 15504 que considera que la mejora de procesos de software es un factor esencial para el desarrollo exitoso de soluciones de software, por lo que múltiples organizaciones han adoptado las buenas prácticas de la gestión de servicios para evolucionar a un nivel más competitivo (Mesquida, Mas, Amengual, \& Calvo-Manzano, 2012).

Tabla 1. Escala de evaluación-Servicios de TI

\begin{tabular}{|c|c|c|}
\hline Valoración & $\begin{array}{l}\text { Porcentaje de } \\
\text { Implementación }\end{array}$ & Evaluación \\
\hline 0 & $00.00 \%$ & $\begin{array}{l}\text { Servicio } \\
\text { Incompleto }\end{array}$ \\
\hline 1 & $\begin{array}{c}{[00.01 \%-} \\
19.99 \%]\end{array}$ & Servicio realizado \\
\hline 2 & $\begin{array}{c}{[20.00 \%-} \\
39.99 \%]\end{array}$ & Servicio gestionado \\
\hline 3 & $\begin{array}{l}{[40.00 \%-} \\
59.99 \%]\end{array}$ & $\begin{array}{l}\text { Servicio } \\
\text { establecido }\end{array}$ \\
\hline 4 & $\begin{array}{c}{[60.00 \%-} \\
79.99 \%]\end{array}$ & Servicio predecible \\
\hline 5 & $\begin{array}{l}{[80.00 \%-} \\
100.00 \%]\end{array}$ & $\begin{array}{l}\text { Servicio de } \\
\text { optimización }\end{array}$ \\
\hline
\end{tabular}

La Tabla 2 presenta la escala de evaluación de los niveles de desarrollo del marco de trabajo de Cobit en las organizaciones. Esta escala de evaluación se basó en los niveles de capacidad y atributos propuesto por la ISO/EIC 15504 que proporciona una base común para realizar evaluaciones de la capacidad del proceso de software, permitiendo el reporte de resultados usando una escala de calificación común (Emam \& Jung, 2001).

Tabla 2. Escala de evaluación-Cobit

\begin{tabular}{ccc} 
Valoración & $\begin{array}{c}\text { Porcentaje de } \\
\text { implementación }\end{array}$ & Evaluación \\
0 & $00.00 \%$ & Inexistente \\
1 & {$[00.01 \%-$} & Muy deficiente \\
& $19.99 \%]$ & Deficiente \\
2 & {$[20.00 \%-$} & Moderado \\
& $39.99 \%]$ & Buena \\
3 & {$[40.00 \%-$} & Muy Buena \\
4 & $59.99 \%]$ & {$[60.00 \%-$} \\
5 & $79.99 \%]$ & \\
5 & {$[80.00 \%-$} & \\
\multicolumn{3}{c}{ Los niveles representan la valoración de la } \\
\end{tabular}

implementación del marco de trabajo de Cobit.
Para recolectar la información respecto al desarrollo del marco de trabajo de Cobit en las organizaciones se utilizó modelos de gestión y gobierno de TI, puesto que permitió la incorporación de un modelo operacional y un lenguaje común a todas las partes de la organización involucradas en actividades de TI. Esto se consideró como uno de los pasos más importantes y críticos hacia un buen gobierno (Isaca, 2012a). La Tabla 3 resume los 37 procesos Cobit que tratan los objetivos de la organización, optimización de riesgos y de los recursos y que han sido considerados para evaluación debido a que agrupan la perspectiva en que la tecnología de la información, gobernanza, gestión de riesgos y cumplimiento son fundamentales en el entorno empresarial actual, ya que la mayoría de los procesos empresariales se basan en la tecnología de la información (Ramalingam, Arun, \& Anbazhagan, 2018).

Tabla 3. Procesos para el Marco de trabajo Cobit

\begin{tabular}{|c|c|}
\hline $\begin{array}{c}\text { Dominio } \\
\text { Cobit }\end{array}$ & Subdominio bajo estudio \\
\hline \multirow{5}{*}{ 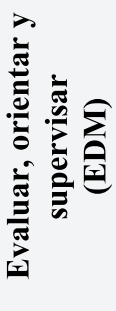 } & $\begin{array}{l}01 \text { Asegurar el establecimiento y } \\
\text { mantenimiento del marco de referencia de } \\
\text { gobierno. }\end{array}$ \\
\hline & 02 Asegurar la entrega de beneficios. \\
\hline & 03 Asegurar la optimización del riesgo. \\
\hline & 04 Asegurar la optimización de recursos. \\
\hline & $\begin{array}{l}05 \text { Asegurar la transparencia hacia las partes } \\
\text { interesadas. }\end{array}$ \\
\hline \multirow{9}{*}{ 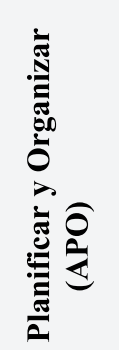 } & 01 Gestionar el marco de gestión de TI. \\
\hline & 02 Gestionar la estrategia. \\
\hline & 03 Gestionar la arquitectura empresarial. \\
\hline & 04 Gestionar la innovación. \\
\hline & 05 Gestionar el portafolio. \\
\hline & 06 Gestionar el presupuesto y los costes. \\
\hline & 07 Gestionar los recursos humanos. \\
\hline & 08 Gestionar las relaciones. \\
\hline & 09 Gestionar los acuerdos de servicio. \\
\hline \multirow{4}{*}{$\stackrel{\Xi}{\rightleftarrows}$} & 10 Gestionar los proveedores. \\
\hline & 11 Gestionar la calidad. \\
\hline & 12 Gestionar el riesgo. \\
\hline & 13 Gestionar la seguridad. \\
\hline \multirow{10}{*}{ 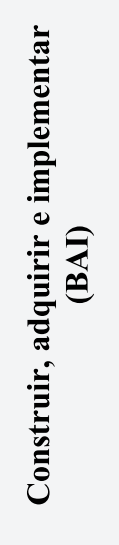 } & 01 Gestionar programas y proyectos. \\
\hline & 02 Gestionar la definición de requisitos. \\
\hline & $\begin{array}{l}\text { 03 Gestionar la identificación y construcción } \\
\text { de soluciones. }\end{array}$ \\
\hline & $\begin{array}{l}\text { 04 Gestionar la disponibilidad y la } \\
\text { capacidad. }\end{array}$ \\
\hline & $\begin{array}{l}05 \text { Gestionar la introducción del cambio } \\
\text { organizativo. }\end{array}$ \\
\hline & 06 Gestionar los cambios. \\
\hline & $\begin{array}{l}07 \text { Gestionar la aceptación del cambio y la } \\
\text { transición. }\end{array}$ \\
\hline & 08 Gestionar el conocimiento. \\
\hline & 09 Gestionar los activos. \\
\hline & 10 Gestionar la configuración. \\
\hline
\end{tabular}




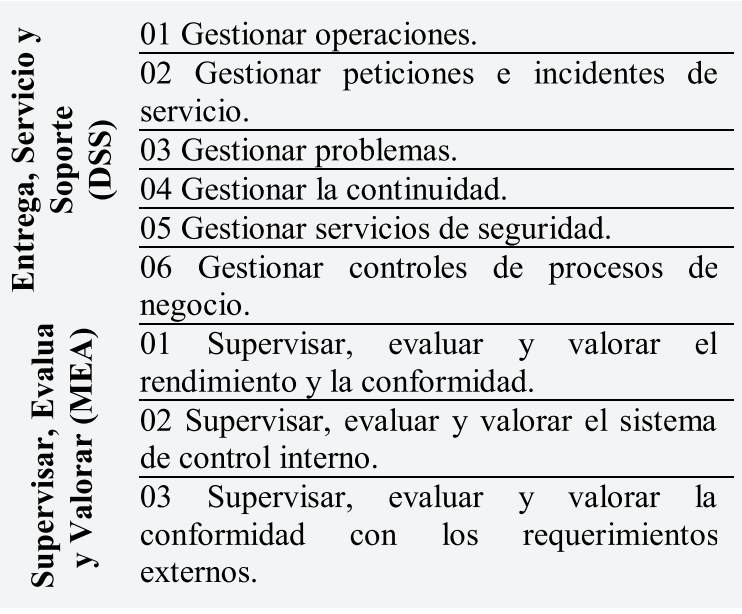

La investigación consideró las fases inicial y final, con el fin de demostrar el cambio entre los niveles madurez de servicios de TI involucrados en las organizaciones. En la fase inicial se realizó una investigación de campo cuantitativa mediante la aplicación de listas de comprobación y cuestionarios al personal de TI en las organizaciones bajo estudio. En la fase final se aplicaron nuevamente listas de comprobación y cuestionarios para evaluar la percepción del personal de las organizaciones y el uso del marco de trabajo de Cobit (Santos \& Santos, 2017). Las pruebas estadísticas de Chi-cuadrado de Pearson y Coeficiente de Sperman fueron utilizadas para analizar la relación entre la percepción de los trabajadores del área de TI y el uso del marco de Cobit.

\section{RESULTADOS}

Las buenas prácticas de gobierno corporativo de TI permitieron elevar los niveles de los servicios proporcionados en las organizaciones a través de una mejor gestión de los activos de tecnologías de la información. Sin embargo, en el desarrollo de la investigación se observó un diferente resultado entre las organizaciones públicas y privadas. Mientras, el incremento en organizaciones privadas se hizo muy notorio, en las organizaciones públicas el incremento era mucho más lento. La Tabla 4 resume los cambios identificados entre las etapas de la investigación. El cambio entre los niveles de capacidad de servicio permite evidenciar que la participación de las buenas practicas no llega a fomentar un incremento similar entre organizaciones.
Tabla 4. Escala de evaluación - Servicios de TI Resultados

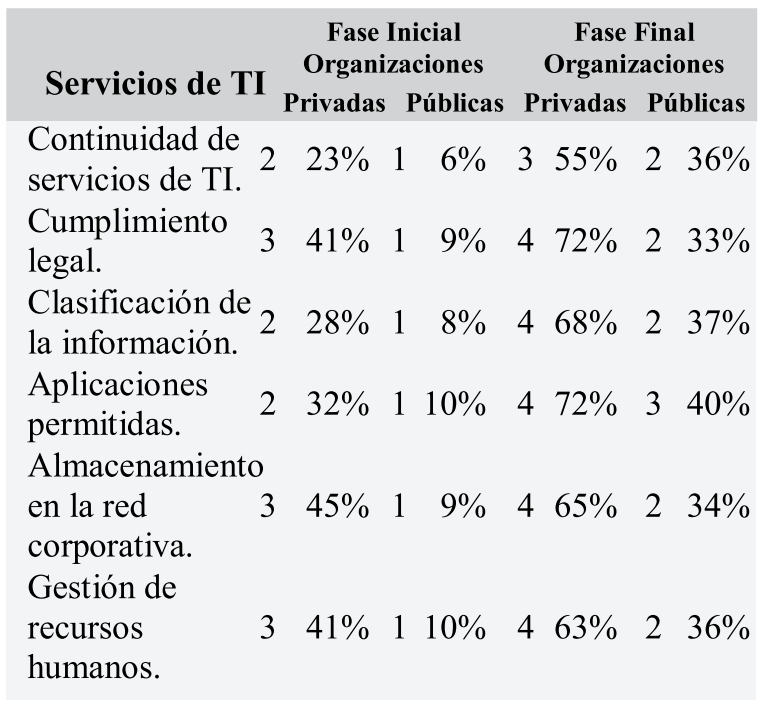

La Figura 4 y la Figura 5 presentan el cambio de nivel entre los puntos de evaluación ante la participación de las buenas prácticas de TI. Para lograr el cambio de nivel fue fundamental el compromiso de la gerencia en las organizaciones y un cambio cultural dentro del personal de las mismas. Esto permitió apreciar el avance o retraso en la ejecución de las estrategias propuestas. Las estrategias propuestas se basaron en:

- La ejecución de planes basados en la gestión de servicios bajo el enfoque de ITIL.

- Adquisición de licencias de diversas herramientas de software necesario para que las organizaciones puedan realizar sus actividades.

- Clasificación y categorización de la información bajo en la perspectiva de Magerit.

- Formulación e implantación de controles de acceso basado en la norma ISO/IEC 27002.

- Formulación e implantación de controles de acceso, almacenamiento en la red y gestión de recursos humanos basados en la norma ISO/IEC 27002.

Al realizar el análisis de la información obtenida se determinó que las organizaciones privadas obtienen en promedio un $31 \%$ de incremento, mientras que las organizaciones públicas han incrementado su capacidad en un $27 \%$. Un análisis basado en solo el incremento numérico se debe evitar debido a que el punto promedio inicial de los servicios de TI en las organizaciones públicas fue del $9 \%$, por lo que su nivel de implementación de servicios fue valorado como nivel 1. Este nivel indicaba que los servicios de TI proporcionados eran catalogados como servicio realizado que cumplían con las metas 
establecidas, pero bajo acciones de manera informal. Este escenario inicial generaba como consecuencia quejas por parte de los usuarios y clientes de la organización. Mientras que el punto promedio inicial de los servicios de TI en las organizaciones privadas fue del $35 \%$, por lo que su nivel de implementación de servicio fue catalogado como nivel 2. Este nivel indicaba que los servicios proporcionados eran catalogados como servicios gestionados, que son controlados y permiten ajustes en su ejecución, sin embargo, no llegaban a proporcionar el valor esperado dentro de la organización.

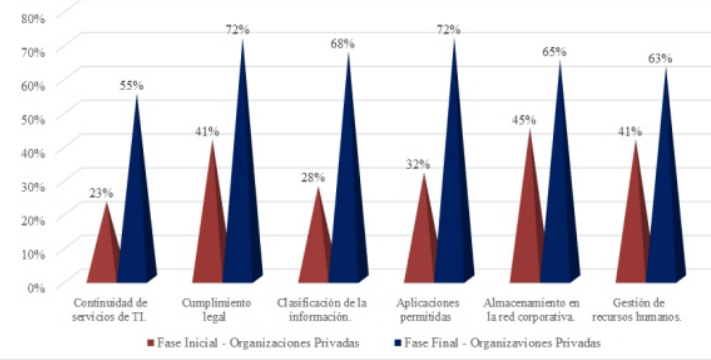

Figura 4. Servicios de TI en organizaciones privadas.

La ejecución de las estrategias de TI basadas en Cobit permitieron incrementar los niveles entre los servicios de TI de las organizaciones. Los servicios de TI en organizaciones públicas lograron alcanzar un promedio de $36 \%$ de implementación, por lo que ahora alcanzan un nivel de 2 que les permiten ser catalogados como servicios gestionados que ahora permiten control $\mathrm{y}$ ajustes en su ejecución. Mientras que los servicios de TI en organizaciones privadas lograron alcanzar un promedio de $66 \%$ de implementación, por lo que ahora alcanzan un nivel de 3 que les permiten ser catalogados como servicios predecibles que operan dentro de límites establecidos a través de un control que permite medir su rendimiento en el cumplimiento de los objetivos de la organización.

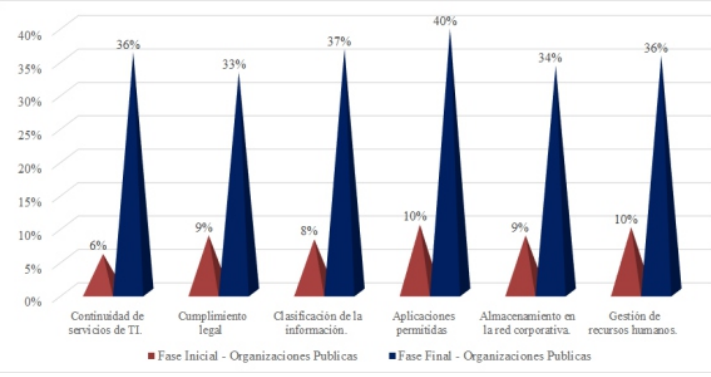

Figura 5. Servicios de TI en organizaciones públicas.

La Tabla 5 resume los cambios identificados entre las etapas de la investigación. El cambio entre los niveles de desarrollo del marco de trabajo de Cobit en las organizaciones permite establecer que las organizaciones públicas y privadas no guardan una relación similar de evolución.

Tabla 5. Procesos para el Marco de trabajo Cobit

\begin{tabular}{|c|c|c|c|c|c|}
\hline \multirow{2}{*}{ Servicios de TI } & \multicolumn{3}{|c|}{$\begin{array}{c}\text { Fase Inicial } \\
\text { Organizaciones }\end{array}$} & \multicolumn{2}{|c|}{$\begin{array}{c}\text { Fase Final } \\
\text { Organizaciones }\end{array}$} \\
\hline & & rivadas & Públicas & Privadas & Públicas \\
\hline $\begin{array}{l}\text { Continuidad de } \\
\text { servicios de TI. }\end{array}$ & 2 & $23 \%$ & $16 \%$ & $355 \%$ & $236 \%$ \\
\hline $\begin{array}{l}\text { Cumplimiento } \\
\text { legal. }\end{array}$ & 3 & $41 \%$ & $19 \%$ & $472 \%$ & $233 \%$ \\
\hline $\begin{array}{l}\text { Clasificación de } \\
\text { la información. }\end{array}$ & 2 & $28 \%$ & $18 \%$ & $468 \%$ & $237 \%$ \\
\hline $\begin{array}{l}\text { Aplicaciones } \\
\text { permitidas. }\end{array}$ & 2 & $32 \%$ & $110 \%$ & $472 \%$ & $340 \%$ \\
\hline $\begin{array}{l}\text { Almacenamiento } \\
\text { en la red } \\
\text { corporativa. }\end{array}$ & 3 & $45 \%$ & $19 \%$ & $465 \%$ & $234 \%$ \\
\hline $\begin{array}{l}\text { Gestión de } \\
\text { recursos } \\
\text { humanos. }\end{array}$ & 3 & $41 \%$ & $110 \%$ & $463 \%$ & $236 \%$ \\
\hline
\end{tabular}

La Figura 6 y la Figura 7 presentan un cambio en el desarrollo del marco de trabajo de Cobit. La participación de la gerencia impulsó la ejecución de las fases de implementación del ciclo de vida de Cobit debido a que permite establecer un proceso sistemático para garantizar que todas las partes interesadas estén preparadas y comprometidas con los cambios que implica pasar de un estado actual a un futuro estado deseado (Isaca, 2012b). Las estrategias se centraron en los siguientes puntos:

- Identificar requisitos y objetivos de las partes interesadas bajo un consenso sobre el enfoque $\mathrm{y}$ alcance.

- Formular una evaluación abierta y justa de las actividades de TI.

- Evaluar la formulación de planes de mejora e iniciativas prioritarias relacionadas con objetivos de la organización.

- Coordinar acciones para asegurar la viabilidad y razonabilidad de la planificación a través de la asignación de recursos.

- Asegurar la ejecución de proyectos de TI a través de la coordinación con los dueños de procesos involucrados y un adecuado control de riesgos.

- Realizar acciones de retroalimentación desde la supervisión de proyectos basados en la criticidad.

- Mantener el liderazgo y crear políticas, normas y procesos que garanticen la gobernabilidad en la organización.

La evaluación del desarrollo del marco de trabajo de Cobit permitió establecer que las 
organizaciones privadas lograron un promedio de $39.20 \%$ de evolución, mientras que las organizaciones públicas lograron un promedio de $17.60 \%$. Las organizaciones públicas tuvieron un punto promedio inicial de $9 \%$ en el marco de desarrollo de Cobit, mientas que las organizaciones privadas tuvieron un punto inicial de $15 \%$. En ambos tipos de organización se estableció como punto inicial el nivel $1-M u y$ deficiente, que evidenciaba que los procesos de TI no eran adecuadamente tratados o gestionados, es decir, que los proceso de Cobit no se encontraban formalmente establecidos o en algunos casos eran inexistentes en las organizaciones.

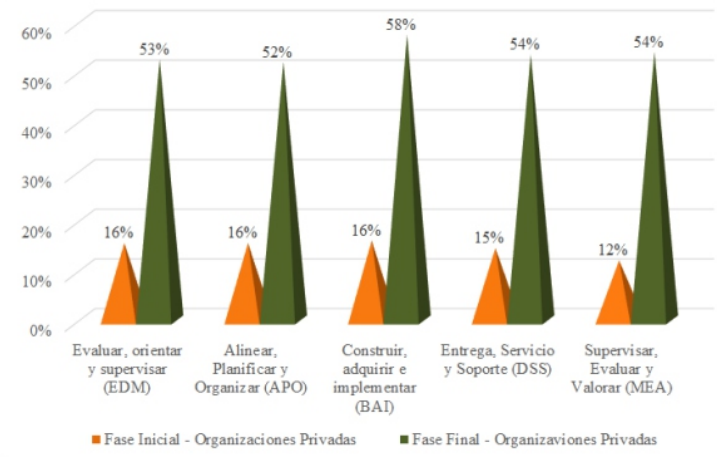

Figura 6. Marco de trabajo de Cobit en organizaciones privadas.

La participación de la gerencia y el cambio cultural del personal de las organizaciones lograron el cambio en beneficio de la organización. Luego de ejecutar las estrategias basadas en la gestión de servicios de TI se logró una evolución favorable en el marco de desarrollo de Cobit. Las organizaciones privadas lograron alcanzar un promedio de $39.20 \%$ de evolución que ha permitido ubicar a estas organizaciones en un $54 \%$ de desarrollo del marco de trabajo de Cobit y en un nivel de 3 -Moderado, que indica que cuenta con la capacidad de lograr los objetivos formulados. Mientras que las organizaciones públicas lograron una evolución de $17.60 \%$, que ha permitido ubicar a estas organizaciones en un $27 \%$ y en un nivel $2-$ Deficiente, que indica que aún no se cuenta con un desarrollo del marco de trabajo de Cobit adecuado.

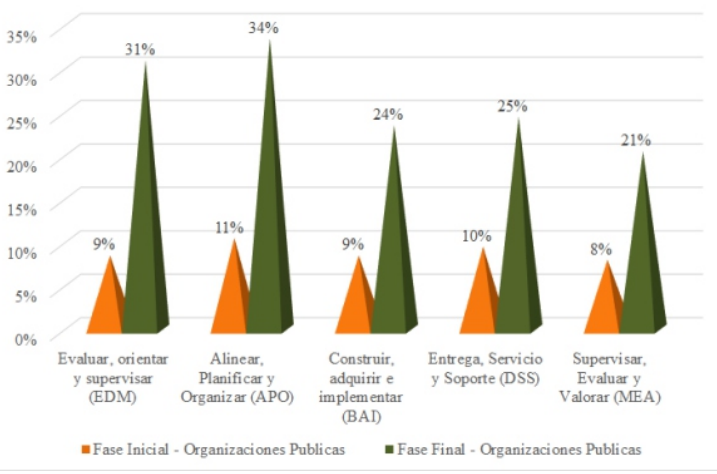

Figura 7. Marco de trabajo de Cobit en organizaciones públicas.

Se realizó un análisis de la percepción de la gestión de tecnologías de información a través del cumplimiento de la guía de referencia de procesos de Cobit en la fase final de la investigación. La Figura 8 resume el comportamiento del personal de tecnologías de información de las organizaciones públicas. En la Figura 8 se aprecia que el $72.88 \%$ del personal percibe que la organización se encuentra en un nivel de $M u y$ Deficiente, un $25.42 \%$ percibe que se encuentra en un nivel de Deficiente y un $1.69 \%$ percibe que la organización se encuentra en un nivel de Moderado.

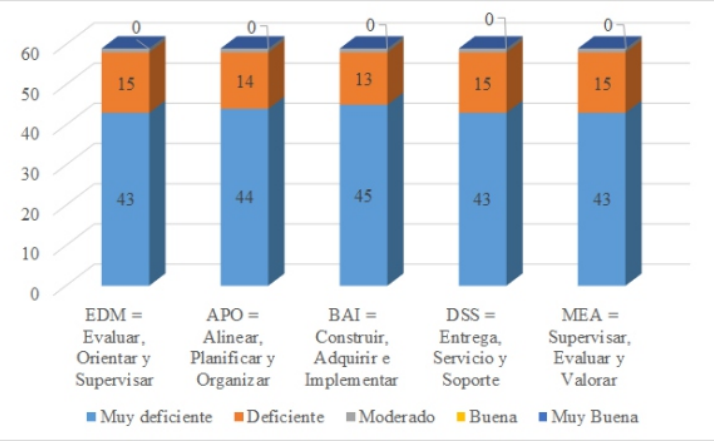

Figura 8. Percepción del uso del marco de Cobit en una organización pública.

La Figura 9 resume el comportamiento del personal de tecnologías de información de las organizaciones privadas. En la Figura 9 se observa que el $17.05 \%$ del personal percibe que la organización se encuentra en un nivel de Muy Deficiente, un $35.15 \%$ percibe que se encuentra en un nivel de Deficiente, un $45.89 \%$ percibe que la organización se encuentra en un nivel de Moderado y un $1.89 \%$ percibe que la organización se encuentra en un nivel de Bueno. 


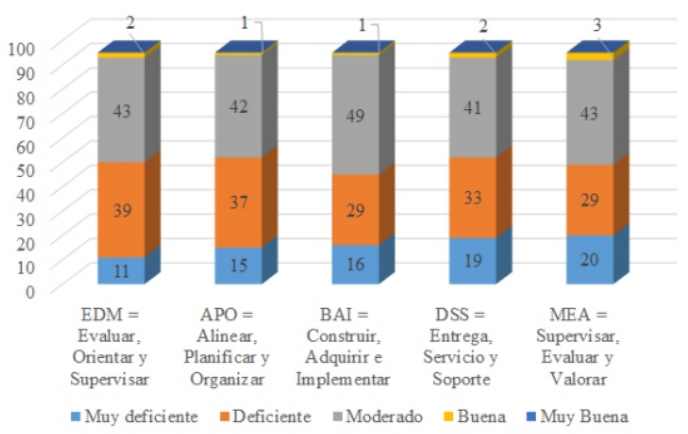

organızación prıvada

Se ha aplicado la prueba estadística $\mathrm{X}^{2}$ para evaluar la asociación de la variable percepción de los trabajadores de tecnologías de información y el uso del marco de Cobit. Se aplicó la evaluación del coeficiente Chi cuadrado de Pearson, el resultado indicó que el p-valor es menor que 0.05 , por lo que la hipótesis de independencia de estas variables se descarta y se acepta la hipótesis que indica que estas dos variables tienen una asociación bajo una organización pública y privada respectivamente.

Se realizó la prueba de normalidad de Shapiro Wilk para los escenarios de organizaciones públicas y privadas. El resultado de la evaluación indicó que el p-valor obtenido es mayor que 0.05 , por lo que se concluye que las muestras no se encuentran normalizadas. Debido a que las muestras no se encuentran normalizadas, se utilizó la prueba estadística de Spearman. En ambos escenarios de estudio se observó que el pvalor obtenido es menor a 0.05 , de ello se concluye que el coeficiente de correlación de Spearman fue significativo. Los valores de los coeficientes fueron positivos, por lo que se concluye que la correlación es positiva y directa. Por lo que, a una mejor percepción de los trabajadores de tecnologías de información, le corresponderá un mejor valor en el grado de uso del marco de trabajo de Cobit.

Se encontró que los coeficientes de correlación de Spearman fueron de 0.963 y 0.778 para las organizaciones públicas y privadas respectivamente. De estos valores se puede concluir que el nivel de correlación es moderado a fuerte puesto que se acercan al valor de 1 .

\section{DISCUSIÓN}

Existe una coincidencia en la naturaleza de la relación entre el gobierno de tecnologías de información y diversos factores como: El alineamiento de negocios/tecnologías de información, entrega de valor de tecnologías de información, administración de recursos de tecnologías de información, administración de riesgos de tecnologías de información y rendimiento de tecnologías de información (Alkhaldi, Hammami, \& Uddin, 2017). Estos factores organizados en categorías tienen impacto directo con el personal de tecnologías de información y de la gerencia, por lo que una estructura de evaluación de rápida comprensión es una gran ventaja durante el proceso de recopilación de información en una investigación.

El gobierno de tecnologías de información adopta diversas estrategias para su despliegue en la organización: nivel de procesos, sistemas de información integrados, sistemas de apoyo y nivel de tecnología (Velásquez, Puentes, \& Pérez, 2015). Este criterio ha sido sumamente útil al momento de capacitar al personal de la organización para que logre adoptar el uso de indicadores de evaluación del uso del marco de trabajo de Cobit. El marco de trabajo de Cobit asociado con estrategias de despliegue de gobierno de tecnologías de información permite una rápida identificación de los procesos débiles de la organización y permitir oportunidades de mejora dentro de la misma.

Sin embargo, existen áreas de desarrollo de las tecnologías de información que se ven afectadas por bajos niveles de gobierno de TI. Esta falta de gobernabilidad viene provocando una desaceleración en el proceso de implementación del marco de trabajo de Cobit como solución de gobierno de TI. Si este problema se llegase a presentar en una organización se recomienda adoptar modelos de tecnologías de información respaldados en ITIL, Cobit e ISO/IEC 27001, debido a que estos modelos se han desarrollado para diferentes propósitos y aspectos del gobierno de tecnologías de información '(Bounagui, Mezrioui, \& Hafiddi, 2019). Esta investigación en sus actividades iniciales adoptó el análisis de modelos. Sin embargo, esto generó puntos de vistas y opiniones en diversas direcciones que poco ayudaron a la investigación por lo que la adopción del modelo de marco de trabajo de Cobit es una buena alternativa para equilibrar conceptos y ejecutar planes de acción en la organización.

Una relación directa se establece entre el nivel de madurez y la aplicación de un modelo de Cobit. Sin embargo, se debe tener presente que la selección de diversas fuentes como elementos clave puede llevar a resultados diferentes. Una estrategia para validar las selección es una combinación entre el instrumento utilizado para recopilar información y entrevistar al personal 
involucrado (Smits \& van Hillegersberg, 2017). Esta estrategia fue utilizada para evidenciar la asociación y correlación entre la percepción y uso del marco de trabajo de Cobit en una organización. No debe considerarse esta relación directa o absoluta por lo que es razonable proponer mejora en los instrumentos propuestos en el estudio.

Se debe tener en consideración que Cobit no llega a cumplir con estándares gubernamentales para proyectos de inversión social y requerimientos de la gestión pública (Zhi \& Zhou, 2013). Muchas organizaciones públicas consideran que Cobit no se adapta a las necesidades sociales de una comunidad, debido a que suelen considerar que un proyecto social es diferente a un proyecto empresarial. Cobit promueve el gobierno y gestión de TI. La generación de valor es un principio fundamental y no se limita al tipo de proyecto en ejecución.

Diversas herramientas de tecnologías de información son puestas en producción en organizaciones. Cobit se ha venido utilizando como marco para identificar procesos de tecnologías de información y para analizar riesgos involucrados en las soluciones de TI. Las actividades de recolección de datos e información se obtienen a través de herramientas de entrevistas y observación (Astuti et al., 2017). E1 procesamiento de la información ayudará a los responsables a una adecuada toma de decisiones estratégicas, al mismo tiempo que esta documentación será útil como caso de éxito para diversas organizaciones.

La tecnología de la información se ha vuelto esencial para apoyar el crecimiento y la sostenibilidad de todo tipo de organizaciones. El proceso de identificar los mecanismos correctos para un contexto específico es un esfuerzo complejo (Scalabrin Bianchi \& Dinis Sousa, 2016). Asimismo, el comportamiento de las organizaciones ha migrado al uso de las tecnologías de información en diversas áreas, cuyo punto inicial se ha basado en la gestión de procesos para luego dar paso a la gestión de servicios de TI. Por lo Cobit se integra satisfactoriamente como mecanismo de control y supervisión.

La información y el conocimiento están involucrados directamente en los procesos. El gobierno de tecnologías de información establece sólidas prácticas para una organización. El gobierno de tecnologías de información gestiona de manera eficiente y efectiva los recursos de tecnologías de información (Suicimezov \& Radu Georgescu, 2014). La medición del éxito de los procesos se relaciona directamente con el grado de eficiencia y eficacia de la información generada. Los trabajadores de tecnologías de información llegar a percibir directamente el grado de uso del marco de trabajo de Cobit. Esta participación activa de los trabajadores como principal activo de la organización se convierte en un catalizador que eleva el grado de logro de metas en la organización.

El gobierno corporativo requiere de la participación del personal de la organización debido a la directa asociación que mantiene con la gestión del conocimiento que se requiere para su implementación (Bin-Abbas \& Bakry, 2014). El personal de las organizaciones llega a percibir cambios en los procesos establecidos en las organizaciones. Estos cambios de forma se llegan a apreciar mucho mejor en organizaciones privadas debido a que estas se establecen en el cumplimiento de metas, lo que se aprecia poco en organizaciones públicas debido a su dificultad en la implementación de Cobit, producto de la dificultad de establecer un cambio cultural.

\section{CONCLUSIONES}

Existe una relación directa y fuerte entre la percepción de los trabajadores y el uso del marco de trabajo de Cobit. Los usuarios internos y externos a la organización perciben esto basándose en la ejecución de los procesos y servicios de tecnologías de información más agiles, más controlados y encaminados en relación con las políticas de la organización. De igual manera, las herramientas de recolección de información deben ser actualizadas y revisadas periódicamente a fin de garantizar la mejor fuente de información.

Se ha apreciado que las organizaciones privadas desarrollan, en mejor medida, el marco de gobierno corporativo de tecnologías de información a comparación de una organización pública. Esto se viene generando debido a las políticas y metas establecidas, identificación del valor de las tecnologías de información y el control de riesgos de las tecnologías de 
información en las organizaciones. Del mismo modo, la implantación de un cambio organizacional basado en el marco de trabajo de Cobit ha logrado establecer conceptos homogéneos que han mejorado las líneas de comunicación entre áreas.

Las buenas prácticas de ITIL aplicadas entre los activos de TI han permitido una rápida integración con el marco de trabajo de Cobit. El uso de ITIL ha favorecido a mejorar la gestión de activos de TI que fue un buen insumo para el desarrollo del marco de trabajo de Cobit en las organizaciones bajo estudio.

\section{REFERENCIAS}

ALKHALDI, Firas; HAMMAMI, Samir \& UDDIN, Mohammed. Understating value characteristics toward a robust IT governance application in private organizations using COBIT framework. International Journal of Engineering Business Management, 9, 1-8, 2017. https://doi.org/10.1177/1847979017703779

ALREEMY, Z., CHANG, V., WALTERS, R., \& WILLS, G. (2016). Critical success factors (CSFs) for information technology governance (ITG). International Journal of Information Management, 36(6): 907- 916, $2 \quad 0 \quad 1 \quad 6$ https://doi.org/10.1016/j.ijinfomgt.2016.05. 017

AstuTi, H. M., MUQTADIROH, F. A., DARMANINGRAT, E. W. T., \& PUTRI, C. U. Risks Assessment of Information Technology Processes Based on COBIT 5 Framework: A Case Study of ITS Service Desk. Procedia Computer Science, 124, 569576 , 20017 https://doi.org/10.1016/j.procs.2017.12.191

BIN-ABBAS, H., \& BAKRY, S. H. Assessment of IT governance in organizations: A simple integrated approach. Computers in Human B ehavior, 32, $261-267,2014$. https://doi.org/10.1016/j.chb.2013.12.019

BOUNAGUI, Y., MEZRIOUI, A., \& HAFIDDI, H. Toward a unified framework for Cloud Computing governance: An approach for evaluating and integrating IT management and governance models. Computer Standards and Interfaces, 62, 98-118, 2019. https://doi.org/10.1016/j.csi.2018.09.001

EMAM, K. El, \& JUNG, H.-W. An empirical evaluation of the ISO/IEC 15504 assessment model. Journal of Systems and Software, $59(1), \quad 23-41, \quad 2001$. https://doi.org/10.1016/S01641212(01)00046-2

GA N T M N , S., \& F E D OROW I C Z, J .
Communication and control in outsourced IS development projects: Mapping to COBIT domains. International Journal of Accounting Information Systems, 21, 63-83, 20106 https://doi.org/10.1016/j.accinf.2016.05.001

GËRVALLA, M., PRENIQI, N., \& KOPACEK, P. IT Infrastructure Library (ITIL) framework approach to IT Governance. IFACPapersOnLine, 51(30), 181-185, 2018. https://doi.org/10.1016/j.ifacol.2018.11.283

HUSE, M. Accountability and Creating Accountability: a Framework for Exploring Behavioural Perspectives of Corporate Governance. British Journal of Management, 16(s1), S65S79, 2005. https://doi.org/10.1111/j.14678551.2005.00448.x

ISACA. Cobit 5 - Procesos catalizadores. Isaca Copyright. 2012a.

ISACA. Implementation Cobit. EE.UU.: Isaca Copyright. 2012b.

JOSHI, A., BOLLEN, L., HASSINK, H., DE HAES, S., \& VAN GREMBERGEN, W. Explaining IT governance disclosure through the constructs of IT governance maturity and IT strategic role. Information \& Management, 55 ( 3 ), $368-380,2018$. https://doi.org/10.1016/j.im.2017.09.003

JUIZ, C., COLOMO-PALACIOS, R., \& GÓMEZ, B. Cascading ISO/IEC 38500 based Balanced Score Cards to improve board accountability. Procedia Computer Science, $138, \quad 417-424, \quad 2018$. https://doi.org/10.1016/j.procs.2018.10.059

MESQUIDA, A. L., MAS, A., AMENGUAL, E., \& CALVO-MANZANO, J. A. IT service management process improvement based on ISO/IEC 15504: A systematic review. Information and Software Technology,54, $239-247,2012, \mathrm{M}$ a r c h. https://doi.org/10.1016/j.infsof.2011.11.002

R A M A L I N G A M, D ., A R U N, S ., \& ANBAZHAGAN, N. A Novel Approach for Optimizing Governance, Risk management and Compliance for Enterprise Information security using DEMATEL and FoM. Procedia Computer Science, 134, 365-370, $\begin{array}{llll}2 & 0 & 1 & 8\end{array}$ https://doi.org/10.1016/j.procs.2018.07.197

SÁ COUTO, E., FILOMENA CASTRO LOPES, M., \& DINIS SOUSA, R. NC-ND license (http://creativecommons.org/licenses/by-ncnd/4.0/). Peer-review under responsibility of SciKA-Association for Promotion and Dissemination of Scientific Knowledge-NCN D $1 \mathrm{i} \mathrm{c}$ e $\mathrm{n} \mathrm{s}$ (http://creativecommons.org/licenses/by-ncnd/4.0/). Peer-review under responsibility of SciKA-Association for Promotion and Dissemination of Scientific Knowledge. 
Procedia - Procedia Computer Science, 64, 109991106 , 20015 https://doi.org/10.1016/j.procs.2015.08.565

SANTOS, L. C., \& SANTOS, C. D. A study on the impact of non-operational mechanisms on the effectiveness of public information technology governance. Revista de Administração, 52(3): 256267, 2017. https://doi.org/10.1016/j.rausp.2017.05.005

SCALABRIN BIANCHI, I., \& DINIS SOUSA, R. (2016). IT Governance Mechanisms in $\mathrm{H}$ i g h e r E d u c a t i o n. 2016 . https://doi.org/10.1016/j.procs.2016.09.253

SMITS, D., \& VAN HILLEGERSBERG, J. The development of a hard and soft IT governance assessment instrument. Procedia Computer Science, 121, 4754, 2

0 7 https://doi.org/10.1016/j.procs.2017.11.008

SMITS, D., \& VAN HILLEGERSBERG, J. The continuing mismatch between IT governance maturity theory and practice: a new approach. Procedia Computer Science,
$138, \quad 549-560, \quad 2018$. https://doi.org/10.1016/j.procs.2018.10.075 SUICIMEZOV, N., \& RADU GEORGESCU, M. IT Governance in Cloud. Procedia Economics and Finance, 15,830-835, 2014. https://doi.org/10.1016/S22125671(14)00531-0

VELÁSQUEZ, T., PUENTES, A., \& PÉREZ, Y. (2015). Un enfoque de buenas prácticas de gobierno corporativo de TI. Tecnura, 19, $\begin{array}{llllll}1 & 5 & 9 & 1 & 6 & 9\end{array}$. https://doi.org/10.14483/udistrital.jour.tecnu ra.2015.SE1.a14

ZHI, L., \& ZHOU, X. ScienceDirect The Audit Method Research on Enterprise and Institution Information Technology Projects Invested by Government Selection and peerreview under responsibility of Chinese Overseas Transportation Association (COTA). Procedia-Social and Behavioral Sciences, 96, 1050-1054, 2013. DOI: 10.1016/j.sbspro.2013.08.120 